\title{
Relationship between Delusion of Theft and Cognitive Functions in Patients with Mild Alzheimer's Disease
}

\author{
Hae-Ran $\mathrm{Na}^{1}$, Dong Woo Kang', Young-Sup Woo², \\ Won-Myong Bahk ${ }^{2}$, Chang-Uk Lee ${ }^{1}$, and Hyun Kook Lim ${ }^{2} \bowtie$ \\ 1'Department of Psychiatry, Seoul St. Mary's Hospital, College of Medicine, The Catholic University of Korea, Seoul, Republic of Korea \\ ${ }^{2}$ Department of Psychiatry, Yeoui-do St. Mary's Hospital, College of Medicine, The Catholic University of Korea, Seoul, Republic of Korea
}

\begin{abstract}
Although delusion of theft (DT) is the most frequent type of delusion in Alzheimer's disease (AD), its relationship to cognitive functions remains unclear. The aim of this study was to demonstrate the relationship between DT and cognitive functions in mild AD. Two hundred eighty-nine mild $\mathrm{AD}$ patients were enrolled in this study. These subjects were classified into three groups: patients with no delusions (ND, $\mathrm{n}=82$ ), patients with paranoid delusions $(\mathrm{PD}, \mathrm{n}=114)$ and patients with DT $(\mathrm{n}=93)$. Cognitive functions and their associations with the degree of delusion were compared among the three groups. The results showed that verbal Fluency scores were significantly lower in the PD group than in the DT and ND groups. Word List Recall scores were significantly lower in the DT group than in the PD and ND groups. Interestingly, delusion severity measured with the Neuropsychiatric Inventory delusion subscale correlated negatively with the Word List Recall scores in the DT group. In this study, we demonstrated that episodic memory functions in mild AD patients were associated with DT, but not with PD. Further studies might be needed to clarify the pathophysiology of delusions associated with AD.
\end{abstract}

Psychiatry Investig 2018;15(4):413-416

Key Words Delusion of theft, Cognitive function, Alzheimer's disease.

\section{INTRODUCTION}

Delusion is one of the most common behavioral symptoms associated with Alzheimer's disease (AD), disturbing patient quality of life and cognitive functions. ${ }^{1,2}$ The prevalence of delusion in $\mathrm{AD}$ has been reported to be about $36 \%$, ranging from $9.3 \%$ to $63 \%{ }^{3,4}$ Among various kinds of delusions, delusion of theft (DT) is the most frequent type in $\mathrm{AD} .^{5}$ Several prior studies investigated the relationship between cognitive functions and $\mathrm{DT}$ in $\mathrm{AD}$, but the results remain inconclusive. Although not specified for DT, some studies reported that delusion correlated significantly with cognitive function test scores such as the Mini Mental State Examination (MMSE), ${ }^{6}$ whereas other studies reported that there was no significant correlation. ${ }^{7}$

Received: May 10, 2017 Revised: July 10, 2017

Accepted: September 20, 2017

$\triangle$ Correspondence: Hyun Kook Lim, MD, PhD

Department of Psychiatry, Yeoui-do St. Mary's Hospital, College of Medicine, The Catholic University of Korea, 10 63-ro, Yeongdeungpo-gu, Seoul 07345, Republic of Korea

Tel: +82-2-3779-1048, Fax: +82-2-780-6577, E-mail: drblues@catholic.ac.kr

(a) This is an Open Access article distributed under the terms of the Creative Commons Attribution Non-Commercial License (http://creativecommons.org/licenses/by$\mathrm{nc} / 4.0$ ) which permits unrestricted non-commercial use, distribution, and reproduction in any medium, provided the original work is properly cited.
The limitations of previous studies have mostly resulted from their small sample sizes and lack of solid longitudinal data. Moreover, in previous studies, DT may not have been completely distinguished from other kinds of paranoid delusion (PD).

The aim of this study was to determine the relationship between cognitive functions and $\mathrm{DT}$ in mild $\mathrm{AD}$. To overcome the aforementioned limitations, we collected a reasonablysized sample of subjects and compared the cognitive function patterns of $\mathrm{AD}$ subjects with $\mathrm{DT}$ and $\mathrm{PD}$.

\section{METHODS}

Two hundred eighty-nine patients who visited St. Vincent's Hospital were recruited for this study. The inclusion criteria were 1) a diagnosis of probable AD according to the National Institute of Neurological and Communicative Disorders and Stroke (NINCDS) and the Alzheimer's disease and Related Disorders Association (ADRDA) criteria ${ }^{8}$ and 2) a score on the Clinical Dementia Rating Scale $(\mathrm{CDR})=1$. $^{9}$ Subjects who had other neurological or psychiatric conditions were excluded. The Korean version of the Consortium to Establish A Registry for Alzheimer's Disease (CERAD-K) neuropsycho- 
logical battery, ${ }^{10}$ including Verbal Fluency (VF), 15-item Boston Naming Test (BNT), MMSE, Word List Memory (WLM), Word List Recall (WLR), Word List Recognition (WLRc), Constructional Praxis (CP), and Constructional Recall (CR), was used to assess cognitive functions. In addition, the Neuropsychiatric Inventory (NPI) ${ }^{11}$ was used to assess the severity of behavioral symptoms. This study was approved by the Institutional Review Board of the Catholic University of Korea. Written informed consent was obtained from all subjects and their guardians (IRB No. VC15EISI0044).

Study participants were classified into three groups according to the presence and type of delusion: $\mathrm{AD}$ with no delusions (ND), AD with PD (the false belief that one is being harmed or persecuted by a particular person or group of people) and $\mathrm{AD}$ with DT (the false belief that someone has stolen something). Most of paranoid themes were related with 'my family (someone) is trying to harm or kill me.' One of the contents was "My daughter-in-law seems to have poisoned my rice." DT includes 'my daughter stole my jewelry' or 'my son seems to have taken my pocket money'. We excluded subjects who had multiple types of delusions. The severity of delusions was measured with the NPI delusion subscale.

Statistical analyses of demographic data were performed with the Statistical Package for the Social Sciences (SPSS, version 12.0, SPSS Inc., Chicago, IL, USA). The assumption of normality was tested for all continuous variables with the
Kolmogorov-Smirnov test. All variables were normally distributed except the NPI delusion subscale scores. The independent $\mathrm{t}$ - and $\chi^{2}$ tests were used to assess potential differences among the three groups for all demographic variables. In addition, the Mann-Whitney U test was used to compare the group differences in the NPI delusion subscale between the PD and the DT groups.

Analysis of covariance (ANCOVA) and post hoc tests using Tukey's multiple comparison tests were performed to identify group differences in cognitive functions after controlling for age, education and gender effects. In addition, as the NPI delusion subscale value of the each group was not normally distributed, the non-parametric Spearman correlation analysis was used to identify the association pattern between cognitive functions and the degree of delusion in the PD and DT groups.

For all statistical analyses, a two-tailed p-value less than 0.05 was considered statistically significant.

\section{RESULTS}

\section{Demographic data}

There were no significant differences in baseline demographic characteristics among the three groups (Table 1). There were no differences in NPI scores between the PD and DT groups.

Table 1. Demographic and clinical characteristics of the study participants

\begin{tabular}{|c|c|c|c|c|}
\hline & $\mathrm{ND}(\mathrm{N}=82)$ & $\mathrm{PD}(\mathrm{N}=114)$ & $\mathrm{DT}(\mathrm{N}=93)$ & $\mathrm{p}$ value \\
\hline Age (years $\pm \mathrm{SD})$ & $76.9 \pm 6.5$ & $78.4 \pm 6.0$ & $77.5 \pm 6.7$ & NS \\
\hline Education (years $\pm S D$ ) & $7.8 \pm 2.9$ & $7.9 \pm 2.9$ & $7.6 \pm 3.2$ & NS \\
\hline $\operatorname{Sex}(M: F)$ & $28: 54$ & $27: 87$ & $29: 64$ & NS \\
\hline CDR & 1 & 1 & 1 & NS \\
\hline CDR-SB & $4.0 \pm 3.7$ & $4.1 \pm 2.9$ & $4.1 \pm 3.1$ & NS \\
\hline S-IADL & $19.8 \pm 5.5$ & $19.5 \pm 6.4$ & $19.2 \pm 6.8$ & NS \\
\hline S-ADL & 0 & 0 & 0 & NS \\
\hline \multicolumn{5}{|l|}{ CERAD-K battery (SD) } \\
\hline VF & $6.6 \pm 3.2$ & $5.4 \pm 2.6$ & $7.4 \pm 3.6$ & $<0.001$ \\
\hline BNT & $5.6 \pm 2.7$ & $5.2 \pm 2.8$ & $6.0 \pm 2.9$ & NS \\
\hline MMSE & $20.8 \pm 3.6$ & $20.0 \pm 3.6$ & $20.5 \pm 2.5$ & NS \\
\hline WLM & $7.2 \pm 3.6$ & $6.8 \pm 3.6$ & $6.5 \pm 2.1$ & NS \\
\hline $\mathrm{CP}$ & $7.2 \pm 2.2$ & $7.1 \pm 2.2$ & $7.5 \pm 2.3$ & NS \\
\hline WLR & $1.7 \pm 1.1$ & $1.8 \pm 1.6$ & $0.9 \pm 1.4$ & $<0.001$ \\
\hline WLRc & $3.2 \pm 2.6$ & $3.7 \pm 3.1$ & $3.5 \pm 2.9$ & NS \\
\hline CR & $1.0 \pm 1.4$ & $1.0 \pm 1.7$ & $0.9 \pm 1.6$ & NS \\
\hline
\end{tabular}

ND: AD without any delusions, PD: AD with paranoid delusion, DT: AD with delusion of theft, SD: standard deviation, NS: not significant, CDR: clinical dementia rating scale, CDR-SB: sum of box of the clinical dementia rating scale, S-IADL: Seoul instrumental activities of daily living, S-ADL: Seoul basic activities of daily living, CERAD-K: Korean version of Consortium to Establish A Registry for Alzheimer's disease, VF: verbal fluency, BNT: 15-item Boston Naming Test, MMSE: Mini Mental State Examination, WLM: word list memory, CP: constructional praxis, WLR: word list recall, WLRc: word list recognition, CR: constructional recall 


\section{Cognitive functions and delusions}

Figure 1 and Table 1 present group differences in cognitive function scores. The VF $(\mathrm{p}<0.001)$ and WLR $(\mathrm{p}<0.001)$ scores differed significantly among the three groups. Post hoc analysis revealed that the VF scores of the PD group were significantly lower than those of the DT ( $\mathrm{p}<0.001)$ and ND $(\mathrm{p}=0.02)$ groups. The WLR scores of the DT group were significantly lower than those of the $\mathrm{PD}(\mathrm{p}<0.001)$ and $\mathrm{ND}(\mathrm{p}=0.004)$ groups. On the other hand, WLR scores did not differ significantly between the ND and PD groups. Interestingly, WLR (Spearman's rho $=-0.23, \mathrm{p}=0.026$ ) and CR (Spearman's $r h o=-0.21$, $\mathrm{p}=0.045$ ) scores correlated negatively with delusion severity measured by the NPI delusion subscale in the DT group. However, there were no significant relationships between cognitive functions and the degree of delusion in the PD group.

\section{DISCUSSION}

To the best of our knowledge, this study is the first to demonstrate distinctive association patterns between DT and episodic memory disturbances in mild $\mathrm{AD}$ patients. The strength of our study resulted from our recruitment of a relatively large sample and our exclusion of subjects with co-existing multiple delusions.

In this study, we found that WLR scores were significantly lower in the DT group than in the other two groups. In addition, delusion severity correlated negatively with WLR/CR scores in the DT group. These relationships might be attributable to the memory-associated nature of DT itself (i.e., the belief that someone has stolen something). In other words, DT may be the most common delusion associated with $\mathrm{AD}$ because the primary symptom of $\mathrm{AD}$ is memory impairment. ${ }^{5}$ On the other hand, PD group who had preserved memory functions compared to the theft delusion group, showed decreased verbal fluency. In general, verbal fluency is known to reflect executive function of frontal lobe. It means PD might be related with executive functions perceive or integrate situations rather than the memory impairments. We assume that this difference between two groups might originate from distinct neural substrates. To date, several neuroimaging studies have found that DT is associated with memory-related neuronal substrates in $\mathrm{AD}$ patients. A structural neuroimaging study using computed tomography revealed that right-left medial temporal lobe asymmetry was associated with DT in $\mathrm{AD} .^{12}$ The present study suggests that this asymmetrical pattern might be related to the memory disturbances in $\mathrm{AD}$ patients. Another functional neuroimaging study using SPECT investigated the neuronal substrate for $\mathrm{DT}$ in $\mathrm{AD} .{ }^{13}$ Subjects with DT were found to have significantly lower perfusion in the right medial posterior parietal region (which is known for episodic memory processing) than patients without delusions. Despite the evidence from neuroimaging studies suggesting a distinctive association between episodic memory impairments and DT, few clinical studies have found this association. A previous study identified no significant differences in the scores on Hasegawa's dementia scale (HDS-R), the MMSE or the Neurobehavioral Cognitive Status Examination (COGNISTAT) sub-scales between the DT $(n=14)$ and ND $(n=42)$ groups. ${ }^{14}$ In addition, another study indicated that delayed recall scores did not correlate significantly with the severity

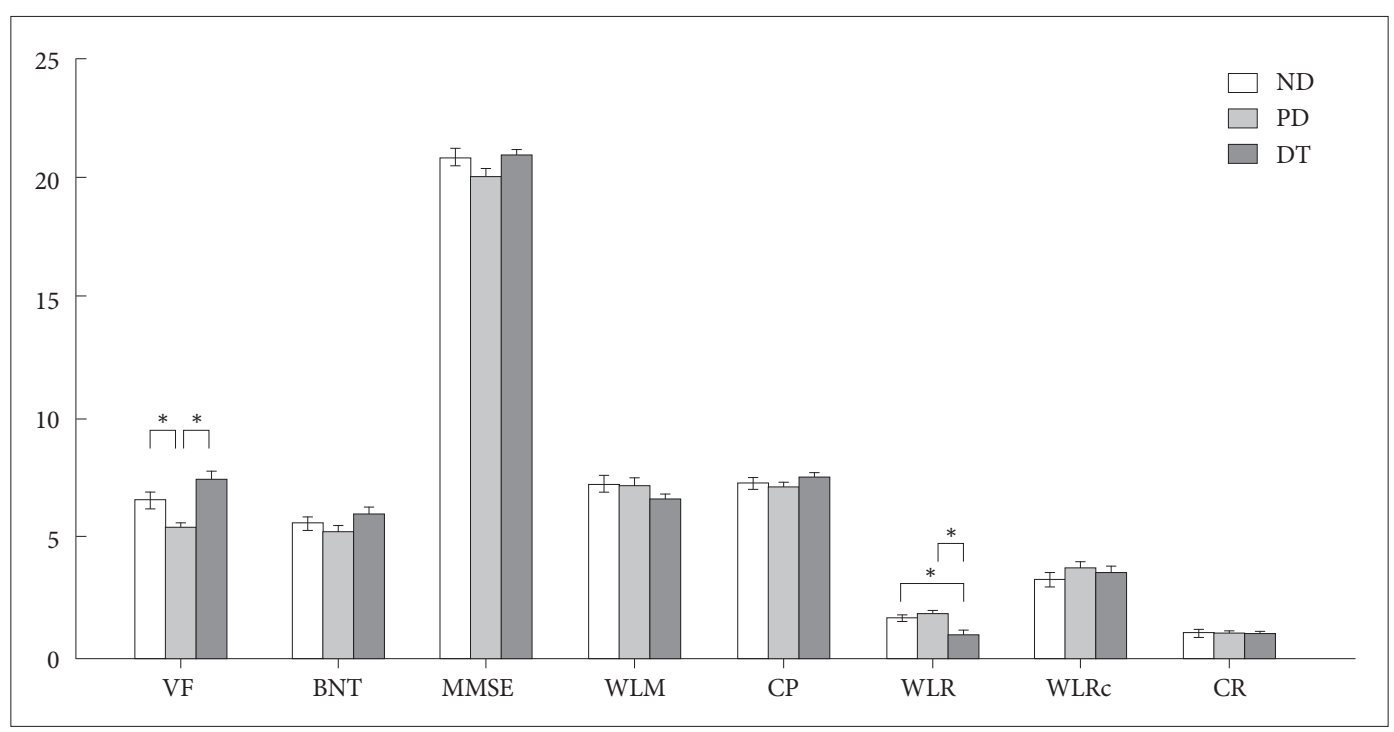

Figure 1. Group differences in cognitive functions measured with the CERAD-K neuropsychological battery. *two-tailed a level of $p<0.05$ for defining statistical significance. ND: AD without any delusions, PD: AD with paranoid delusion, DT: AD with delusion of theft, CERAD-K: Korean version of Consortium to Establish A Registry for Alzheimer's Disease, VF: verbal fluency, BNT: 15-item Boston Naming Test, MMSE: Mini Mental State Examination, WLM: word list memory, CP: constructional praxis, WLR: word list recall, WLRc: word list recognition, CR: constructional recall. 
of DT in $22 \mathrm{AD}$ subjects. ${ }^{15}$ These findings might be attributable to the small sample sizes and the non-exclusion of patients with other types of delusions, such as persecutory, phantom and misidentification delusions.

In this study, we found that the VF scores of the PD group were significantly lower than those of the ND and DT groups. These findings are consistent with previous reports of deficient VF and conceptualization in $\mathrm{AD}$ patients ${ }^{6}$ and of VF and executive functioning deficits in non-demented elderly people with PD. ${ }^{16}$

Several limitations of our study must be taken into consideration. First, to clearly distinguish DT from PD, we could not include $\mathrm{AD}$ patients with mixed delusions and other types of delusions, such as misidentification and phantom delusions. In real clinical settings, mixed delusional $\mathrm{AD}$ patients are more common. Second, as we included subjects with mild-stage $\mathrm{AD}$, our results cannot be generalized to subjects with more severe AD. Larger studies including a broader spectrum of AD subjects would be needed. Third, despite the decent sample size, the NPI delusion subscale value of the each group was not normally distributed. These skewed distributions of the NPI delusion subscale values may affect the association pattern between the NPI delusion subscale and the VF values which were significantly lower than any other group in the PD group. Therefore, regression analysis using normally distributed between the NPI delusion subscales and the cognitive function measures may helpful to predict impact of cognitive dysfunction on delusion severity precisely.

In conclusion, we demonstrated that episodic memory functions in mild $\mathrm{AD}$ patients were associated with DT, but not with PD. Furthermore, mild AD with delusions, regardless of the type, might have a distinct pathophysiology from mild AD without delusions. Further studies are needed to clarify the pathophysiology of delusions associated with AD.

\section{Acknowledgments}

This research was supported by Basic Science Research Program through the National Research Foundation of Korea (NRF) funded by the Ministry of Science, ICT \& Future Planning (NRF-2015R1C1A1A02036578).

\section{REFERENCES}

1. Cipriani G, Danti S, Vedovello M, Nuti A, Lucetti C. Understanding delusion in dementia: a review. Geriatr Gerontol Int 2014;14:32-39.
2. Ornstein K, Gaugler JE, Devanand DP, Scarmeas N, Zhu C, Stern Y The differential impact of unique behavioral and psychological symptoms for the dementia caregiver: how and why do patients' individual symptom clusters impact caregiver depressive symptoms? Am J Geriatr Psychiatry 2013;21:1277-1286.

3. Apostolova LG, Di LJ, Duffy EL, Brook J, Elashoff D, Tseng CH, et al. Risk factors for behavioral abnormalities in mild cognitive impairment and mild Alzheimer's disease. Dement Geriatr Cogn Disord 2014;37: 315-326.

4. Zhao Q-F, Tan L, Wang HF, Jiang T, Tan MS, Tan L, et al. The prevalence of neuropsychiatric symptoms in Alzheimer's disease: systematic review and meta-analysis. J Affect Disord 2016;190:264-271.

5. Holt AE, Albert ML. Cognitive neuroscience of delusions in aging. Neuropsychiatr Dis Treat 2006;2:181-189.

6. Jeste DV, Wragg RE, Salmon DP, Harris MJ, Thal LJ. Cognitive deficits of patients with Alzheimer's disease with and without delusions. Am J Psychiatry 1992;149:184-189.

7. Migliorelli R, Petracca G, Teson A, Sabe L, Leiguarda R, Starkstein SE. Neuropsychiatric and neuropsychological correlates of delusions in Alzheimer's disease. Psychol Med 1995;25:505-513.

8. McKhann GM, Knopman DS, Chertkow H, Hyman BT, Jack CR, Kawas $\mathrm{CH}$, et al. The diagnosis of dementia due to Alzheimer's disease: Recommendations from the National Institute on Aging-Alzheimer's Association workgroups on diagnostic guidelines for Alzheimer's disease. Alzheimer Dement 2011;7:263-269.

9. Morris JC. Clinical dementia rating: a reliable and valid diagnostic and staging measure for dementia of the Alzheimer type. Int Psychogeriatr 1997;9(Suppl 1):173-176.

10. Lee JH, Lee KU, Lee DY, Kim KW, Jhoo JH, Kim JH, et al. Development of the Korean version of the Consortium to Establish a Registry for Alzheimer's Disease Assessment Packet (CERAD-K): clinical and neuropsychological assessment batteries. J Gerontol B Psychol Sci Soc Sci 2002;57:P47-P53.

11. Cummings JL, Mega M, Gray K, Rosenberg-Thompson S, Carusi DA, Gornbein J. The Neuropsychiatric Inventory: comprehensive assessment of psychopathology in dementia. Neurology 1994;44:2308-2314.

12. Geroldi C, Akkawi NM, Galluzzi S, Ubezio M, Binetti G, Zanetti O, et al. Temporal lobe asymmetry in patients with Alzheimer's disease with delusions. J Neurol Neurosurg Psychiatry 2000;69:187-191.

13. Fukuhara R, Ikeda M, Nebu A, Kikuchi T, Maki N, Hokoishi K, et al. Alteration of rCBF in Alzheimer's disease patients with delusions of theft. Neuroreport 2001;12:2473-2476.

14. Murayama N, Iseki E, Endo T, Nagashima K, Yamamoto R, Ichimiya Y, et al. Risk factors for delusion of theft in patients with Alzheimer's disease showing mild dementia in Japan. Aging Ment Health 2009;13: 563-568.

15. Kazui H, Hirono N, Hashimoto M, Nakano Y, Matsumoto K, Takatsuki Y, et al. Symptoms underlying unawareness of memory impairment in patients with mild Alzheimer's disease. J Geriatr Psychiatry Neurol 2006;19:3-12.

16. Ostling S, Johansson B, Skoog I. Cognitive test performance in relation to psychotic symptoms and paranoid ideation in non-demented 85-year-olds. Psychol Med 2004;34:443-450. 